autosomal dominant epilepsy with FS plus, generalized epilepsy with FS plus, and with severe myoclonic epilepsy in infancy that often begins with a prolonged FS. Various $\mathrm{Na}^{+}$ channels and $\mathrm{GABA}_{\mathrm{A}}$ receptors in the brain are probably involved in the pathogenesis of frequent FS and also, in simple FS. Genetic linkage analyses have mapped FS to four loci, FEB1,2,3 and 4, in chromosomes 8q13, 19p, 2q23-q24, and 5q14-q15, respectively. (Hirose S, Mohney RP, Okada M et al. The genetics of febrile seizures and related epilepsy syndromes. Brain Dev August 2003;25:304-312). (Respond: Dr Shinichi Hirose, Department of Pediatrics, School of Medicine, Fukuoka University, 45-1, 7-chome Nanakuma, Jonanku, Fukuoka 814-0180, Japan).

COMMENT. The determination of the molecular genetic mechanism of FS may lead to more specific therapies. FS occur with increased frequency among family members of patients with FS. Tsuboi (1977) reported $17 \%$ of parents and $22 \%$ of siblings of FS probands affected; $30 \%$ of siblings are affected if one parent has a history of FS (Hauser WA. In: Febrile Seizures. Ed by Nelson and Ellenberg, Raven Press, 1981). An analysis of 2,109 patients with FS reported between 1948 and 1963 in 12 different publications showed a mean familial incidence of $17 \%$ (range 2 to $58 \%$ ) (Millichap, 1968).

\title{
GENETICS OF SEVERE MYOCLONIC EPILEPSY OF INFANCY
}

The role of SCN1A gene mutations in the etiology of severe myoclonic epilepsy of infancy (SMEI) was investigated in 93 patients followed at the Hopital Saint Vincent de Paul, Paris, and other centers in France and Italy. SCNIA mutations occurred in 33 patients $(35 \%)$. Parents of three patients $(10 \%)$ who carried the inherited mutations were asymptomatic or had a milder form of epilepsy. Patients with the mutations had a greater frequency of unilateral motor seizures than those without and a more frequent family history of epilepsy. (Nabbout R, Gennaro E, Bernadina BD et al. Spectrum of SCN1A mutations in severe myoclonic epilepsy of infancy. Neurology June (2 of 2) 2003;60:19611967). (Reprints: Dr Rima Nabbout, Hopital Saint Vincent de Paul, Paris, France).

COMMENT. SMEI caused by SCN1A mutations is characterized by unilateral motor seizures, and $10 \%$ of cases are inherited from an asymptomatic or mildly affected parent. SMEI has a mean age of onset of 5 months, more than half the seizures are febrile, seizures are of all types, absence seizures with a myoclonic component are often triggered by photic stimulation, status epilepticus occurs in $75 \%$ and is often precipitated by fever, ataxia develops in $>80 \%$, psychomotor delay is common to all, and seizures are refractory to medication.

\section{EARLY ONSET ABSENCE EPILEPSY}

Neuropsychological evaluations, behavior patterns, and outcomes were studied in a retrospective analysis of 10 patients ( 7 girls, 3 boys) with onset of absence epilepsy before age 3 years, referred between 1986 and 2002 at Hopital Henri Gastaut, Marseille, and Hopital des Enfants, Toulouse, France. At $>2$ years of follow-up (range, 2 years 8 months to 9 years 4 months), only two had typical absences with good seizure control and good cognitive outcome. In the remaining 8 cases, 8 had cognitive delays and behavioral 